\title{
Tracking cardiac engraftment and distribution of implanted bone marrow cells: Comparing intra-aortic, intravenous, and intramyocardial delivery
}

Shu-Hong Li, MD, MSc, ${ }^{a}$ Teresa Y. Y. Lai, MSc, ${ }^{a}$ Zhuo Sun, MD,${ }^{a}$ Mihan Han, BSc, ${ }^{a}$ Eduardo Moriyama, PhD, ${ }^{b}$ Brian Wilson, PhD, ${ }^{b}$ Shafie Fazel, MD, PhD, ${ }^{a}$ Richard D. Weisel, MD, ${ }^{a}$ Terrence Yau, MD, ${ }^{a}$ Joseph C. Wu, MD, PhD, ${ }^{\mathrm{c}}$ and Ren-Ke Li, MD, PhD ${ }^{\mathrm{a}}$

Objectives: Cell therapy improved cardiac function after a myocardial infarction in several preclinical studies; however, the functional benefits were limited in the initial clinical trials, perhaps because of inadequate cell engraftment. We used noninvasive molecular imaging to compare the distribution and myocardial retention of cells implanted by using clinical delivery routes.

Methods: Bone marrow stromal cells isolated from male rats and transfected with a firefly luciferase reporter gene were injected by using 3 increasingly invasive techniques (ie, intravenous, intra-aortic, and intramyocardial) into female rats 3 or 28 days after coronary ligation. Whole-body bioluminescence imaging was performed 2, 24, and 48 hours later; implanted cells were quantified at 48 hours in explanted organs by means of bioluminescence and real-time polymerase chain reaction.

Results: Variations in cell distribution among groups were profound, with nearly complete trapping of the injected cells in the lungs after intravenous delivery. Cell delivery into the aortic root (with the distal aorta occluded) produced minimal cell retention in the heart. Direct intramyocardial injection facilitated the best early targeting of the cells $(P<.05$ vs intravenous and intra-aortic injection). Rapid signal loss over 48 hours indicated very poor cell survival in all 3 groups, although implanted cell retention was greater in mature compared with acute infarcts.

Conclusions: This is the first study to correlate live cell imaging with quantitative genetic and histologic techniques. Noninvasive molecular imaging tracked delivered cells and will permit the evaluation of new and improved delivery platforms designed to increase cell homing, retention, and engraftment.

Supplemental material is available online.

Cell implantation improves cardiac function after a myocardial infarction (MI) in animal studies ${ }^{1-5}$; however, the initial clinical trials did not provide the same benefit. ${ }^{6-9}$ Similarly, cell therapy as an adjunct to coronary bypass surgery produced mixed effects on ventricular function in patients with postinfarction dysfunction. ${ }^{10,11}$ Contributing to these

\footnotetext{
From the Division of Cardiovascular Surgery, ${ }^{\mathrm{a}}$ Toronto General Research Institute, University Health Network, Toronto, Ontario, Canada; the Division of Biophysics and Bioimaging, ${ }^{\mathrm{b}}$ Ontario Cancer Institute, University of Toronto, Toronto, Ontario, Canada; and the Department of Medicine and Radiology, ${ }^{\mathrm{c}}$ Stanford University School of Medicine, Stanford, Calif.

This research was funded primarily by a grant from the Heart and Stoke Foundation of Ontario (T6069) to R-KL and in part by grants from the Canadian Institutes of Health Research (CIHR; MOP14795) to R-KL and from the CIHR (RMF82498). $\mathrm{R}-\mathrm{KL}$ is a Career Investigator of the Heart and Stroke Foundation of Canada and holds a Canada Research Chair in cardiac regeneration. The bioluminescence system was supported by the Canadian Foundation for Innovation.

Received for publication Aug 14, 2008; revisions received Sept 29, 2008; accepted for publication Nov 2, 2008.

Address for reprints: Ren-Ke Li, MD, PhD, MaRS Centre, Toronto Medical Discovery Tower, Room \#3-702, 101 College St, Toronto, Ontario, Canada M5G 1L7 (E-mail: renkeli@uhnres.utoronto.ca).

J Thorac Cardiovasc Surg 2009;137:1225-33

0022-5223/\$36.00

Copyright (c) 2009 by The American Association for Thoracic Surgery

doi:10.1016/j.jtcvs.2008.11.001
}

discrepancies are the timing and mode of cell delivery, which determine implanted cell homing, retention, and extent of engraftment within the damaged myocardium. Low survival rates among implanted cells, as assessed by preclinical and clinical trials, have emphasized the necessity for new approaches to enhance cell engraftment.

Although the quantification of cell survival traditionally relies on postmortem histology, molecular imaging provides an improved opportunity to evaluate new techniques by estimating in vivo survival and engraftment of live cells. In the current study we used a molecular imaging technique to track implanted cells for 48 hours after implantation. ${ }^{12-15}$ Our results provide the first comprehensive comparison of live cell imaging and histologic evidence of postimplantation cell distribution.

Bone marrow stromal cells (BMSCs) injected into the myocardium can engraft in the damaged heart, induce angiogenesis and matrix remodeling, and improve ventricular function. ${ }^{16,17}$ Here, BMSCs transfected with a firefly luciferase (FL) reporter gene were implanted into rats either early or late after an MI by using 3 clinically relevant delivery routes: intra-aortic (cells injected into the aortic root with the distal aorta temporarily occluded to simulate intracoronary delivery), intravenous, or intramyocardial. After implantation, FL is only expressed by live cells. Each living cell with active FL generates a measurable signal in vivo by converting a systemically injected substrate (luciferin) 


$$
\begin{aligned}
& \text { Abbreviations and Acronyms } \\
& \text { Ad-CMV-FL = } \text { firefly luciferase reporter gene } \\
& \text { driven by a constitutive } \\
& \text { cytomegalovirus promoter } \\
&=\text { bone marrow stromal cell } \\
& \text { BMSC } \text { firefly luciferase } \\
& \text { FL }=\text { left anterior descending } \\
& \text { LAD } \text { coronary artery } \\
&=\text { myocardial infarction } \\
& \text { MI }=\text { multiplicity of infection } \\
& \text { MOI }=\text { polymerase chain reaction }
\end{aligned}
$$

to produce photons that can be recorded with a bioluminescent camera and quantified. In contrast, no bioluminescent signals are generated by dead or metabolically inactive (hibernating) cells that cannot convert the substrate.

\section{MATERIALS AND METHODS \\ Experimental Animals}

BMSCs were isolated from male Lewis rats for implantation into female recipients. The Animal Care Committee of the Toronto General Research Institute approved all experimental procedures, which were in accordance with the National Research Council's "Guide for the care and use of laboratory animals", (revised 1996).

\section{Virus Construction and Amplification}

Replication-defective recombinant adenovirus carrying the FL reporter gene driven by a constitutive cytomegalovirus promoter (Ad-CMV-FL) was purchased from Vector Biolabs (Philadelphia, Pa). The viral vector was amplified with AD 293 cells. The titer of the viral stock (supernatant) was determined by using a plaque assay with agarose overlay.

\section{Cell Culture, Transfection, and In Vitro Assay for FL Activity}

Male Lewis rat BMSCs (10-20 $\mu \mathrm{m}$ in diameter, as shown in Figure 1) were cultured ${ }^{18,19}$ and transfected with Ad-CMV-FL at multiplicity of infection (MOI) 10 and 50 for 24 hours. Luciferase assays (BD Biosciences, Mississauga, Ontario, Canada) were performed on days 1, 2, 3, 6, and 7 after viral vector transfection, according to the manufacturer's instructions, to confirm reporter enzyme activity before cell transplantation.

\section{Myocardial Infarction and Cell Transplantation}

Female rats were anesthetized with isoflurane $(2 \%)$ during mechanical ventilation. MI was induced through a thoracotomy by means of occlusion of the left anterior descending coronary artery (LAD). Animals were randomly separated into 6 groups. Rats in each group received $3 \times 10^{6}$ transfected BMSCs in $100 \mu \mathrm{L}$ of serum-free medium at 1 of 2 time points (ie, 3 or 28 days after LAD ligation [LAD3 and LAD28, respectively]) delivered through 1 of 3 routes: intra-aortic, intravenous, or intramyocardial injection. Procedures are detailed in the online-only Materials and Methods section. Three additional groups without LAD ligation (sham controls) also received BMSCs by means of intra-aortic, intravenous, or intramyocardial injection. Therefore cells were delivered to animals in the control, LAD3, and LAD28 groups through 3 different injection routes (ie, intra-aortic, intravenous, and intramyocardial) for a total of 9 groups (see schematic in Figure 2, A).

\section{Optical Bioluminescence Imaging}

At 2, 24, and 48 hours after cell implantation, all rats received an intraperitoneal injection of the FL reporter substrate luciferin (D-luciferin, $375 \mathrm{mg} / \mathrm{kg}$ body weight; Xenogen Corp, Hopkinton, Mass). Live cells expressing the FL enzyme (dead or metabolically inactive cells cannot express FL in vivo) converted the luciferin to inactive oxyluciferin, producing photons that were captured by a charged-coupled device camera system (IVIS, Xenogen Corp). Bioluminescence (generated by viable, metabolically active cells) was quantified in photons per second per square centimeter per steradian. Whole-body imaging was performed for each animal at 48 hours, followed immediately by imaging of explanted organs.

\section{Genomic Real-Time Polymerase Chain Reaction}

Male rat BMSCs were used to generate a standard curve with $\mathrm{Y}$ chromosome copies of $10^{5}, 10^{4}, 10^{3}, 10^{2}$, and $10^{1}$ by using specific primers, as previously described in detail. ${ }^{17}$ Genomic DNA was extracted from excised, homogenized tissues (heart, liver, spleen, kidney and lungs), and Y chromosomes were detected (TaqMan method). The atria and great vessels were removed from the excised hearts before DNA extraction; only the ventricles were included in the analysis.

\section{Immunohistochemistry}

Serial cryosections were stained with a monoclonal anti-luciferase antibody (Santa Cruz Biotechnology, Santa Cruz, Calif) followed by a fluorescein isothiocyanate-conjugated secondary antibody (Molecular Probes, Burlington, Vt) to localize implanted cells. Nuclei were stained with $4^{\prime}-6-$ diamidino-2-phenylindole dihydrochloride (Sigma, Oakville, Ontario, Canada) for in vitro studies or propidium iodide (Sigma) for in vivo studies.

\section{Data Analysis}

All data were expressed as means \pm standard error of the mean. Multiplegroup comparisons were performed by using 1-way analysis of variance. When required, differences were specified by means of post-hoc evaluation with least-squares difference multiple range tests (SPSS software, version 10.0; SPSS, Inc, Chicago, Ill).

\section{RESULTS \\ Effect of FL Reporter Gene Expression on BMSC Viability and Proliferation}

Cultured BMSCs were transfected with Ad-CMV-FL at MOI 10 and MOI 50. We observed no effects of transfection on cell morphology (Figure 1, $A$ and $B$ ) or viability (Trypan Blue exclusion assay: control, $95.6 \% \pm 3.4 \%$; MOI 50 , $92.9 \% \pm 1.7 \%$ ) and no significant effects on cell growth (Figure 1,C).

Immunohistochemical staining with an anti-luciferase antibody showed that more than $90 \%$ of cultured cells were successfully transfected (Figure 1,D-F). Although FL activity was increased (compared with undetectable activity in nontransfected cells) in both groups of transfected BMSCs, the FL activity was nearly 40 -fold higher in cells transfected at MOI 50 compared with those transfected at MOI $10(P<$ .01 ; Figure $1, G$ ). MOI 50 cells were used for the in vivo study. When transfected cells were seeded into culture dishes at different concentrations, bioluminescence counts correlated linearly with cell number $\left(r^{2}=0.99\right.$; Figure 1 , $H$ and $I$ ). 


\section{Systemic Distribution of Implanted Cells (Molecular Imaging)}

The whole-body biodistribution of implanted FL-labeled BMSCs was evaluated in all groups by means of bioluminescence imaging (Figure 2, $B-D$ ). Intra-aortic implantation resulted in diffuse and systemic bioluminescence, with no differences based on the timing of injection. The total signal decreased over time $(P<.05)$, indicating a loss of FL expression likely caused by a decrease in the number of viable cells. Cell retention (compared with the 2-hour time point) decreased by $60 \%$ at 24 hours and by $90 \%$ at 48 hours after cell implantation. Animals that received intravenous cell implantation exhibited bioluminescence confined to the chest at all time points. The signals decreased significantly over time $(P<.05)$, with reductions of $80 \%$ (compared with the 2 -hour time point) by 24 hours and $95 \%$ by 48 hours after implantation. In rats that received intramyocardial cell implantation, bioluminescence was similarly confined to the chest at all time points, but signals were strongest on the left side of the chest, suggesting that cells were mainly localized in the heart. Although bioluminescence decreased over time $(P<.05)$ in the intramyocardial groups, cell retention (compared with the 2-hour time point) was between $30 \%$ and $75 \%$ at 24 hours and remained greater than $25 \%$ at 48 hours after implantation.

At 2 hours after cell implantation, total bioluminescence counts (Figure 2, $E-G$ ) were similar among the 3 implantation routes. At 24 hours, cell retention was lowest when the cells were injected intravenously $(P<.05$ compared with the intra-aortic groups) and highest when the cells were delivered intramyocardially into either acute or chronic scars. At 48 hours after implantation, there were no differences in cell retention between the intra-aortic and intravenous groups, both of which exhibited significantly less retention than the intramyocardial groups $(P<.05$ for all groups). Whole-body bioluminescence counts were statistically similar among groups, irrespective of whether BMSCs were administered at LAD3 or LAD28.

\section{Cell Retention in Tissues (Real-Time Polymerase Chain Reaction and Molecular Imaging)}

The heart, liver, spleen, kidney, and lungs were explanted at 48 hours after cell delivery, imaged immediately by means of bioluminescence, and then subjected to real-time polymerase chain reaction (PCR) quantification (Figure 3). After intra-aortic cell injection, bioluminescence appeared to be distributed primarily within the heart, kidney, and lungs (Figure 3, $A$ and $B$ ). When cells were delivered in an intra-aortic manner into a mature scar (late after the infarct), bioluminescence localized predominantly to the heart, with significantly weaker signals in the remaining organs $(P<.05)$. Consistent with these results, real-time PCR detected the greatest number of implanted cells in the heart when the cells were im- planted in an intra-aortic manner at 28 days after MI $(P<$ .05; Figure 3, $C$ ). Implanted BMSCs were also detected in the kidney and lungs, but not in the liver or spleen, by means of real-time PCR. There was a strong association between results from bioluminescence imaging and real-time PCR after intra-aortic injection.

In the groups that received cells through the intravenous route, both bioluminescence and real-time PCR assays showed that more than $90 \%$ of the surviving cells were trapped in the lungs (Figure 3, $A, D$, and $E$ ). Again, distribution patterns obtained with both methods were in very close agreement.

Intramyocardial injection resulted in bioluminescence signals localized almost entirely to the heart and lungs, with significantly weaker $(P<.05)$ signals in the remaining organs (Figure 3, $A$ and $F$ ). Real-time PCR detected cells only in the heart and lungs (Figure 3, $G$ ), with significantly higher $(P<.05)$ cell retention in the heart than the lungs in all groups-particularly in animals that received cells at 28 days after MI. Imaging revealed a similar pattern (Figure 3, $F$ ), although the differences between signals from the heart and lungs did not reach significance.

\section{Implanted Cell Localization}

Implanted cells were localized by using immunohistochemical staining with an antibody against luciferase protein (Figure 4, A). Results were very similar to those from bioluminescence imaging and real-time PCR studies. In animals that received intra-aortic implantation, donor BMSCs were identified in heart, kidney, and lung tissue, with a few cells also detected in the liver and spleen. In animals that received intravenous implantation, many cells strongly positive for FL were observed in the lungs, with only a few implanted cells present in the other organs. In animals that received intramyocardial implantation, the greatest numbers of donor cells were identified in the heart and lungs.

\section{Cardiac Retention of Cells Implanted Into Acute or Chronic Scars}

Data obtained from isolated hearts were compared among all groups (Figure 4, $B-G$ ). Both bioluminescence imaging and real-time PCR confirmed that maximal cell retention was achieved with intramyocardial implantation. Regarding timing of treatment, although delivery at LAD3 and after the sham operation (control) consistently produced similar results, cell retention after intramyocardial injection into a mature myocardial scar was 6 - to 8 -fold greater $(P<.05)$ than that achieved with intramyocardial injection into control myocardium (Figure 4, $F$ and $G$ ). Similarly, in the intra-aortic group we detected significantly more $(P<.05)$ cells in LAD28 animals than in LAD3 or control animals (Figure $4, B$ and $C)$. Few cells delivered by means of intravenous injection were observed in the myocardial tissue (Figure 4, $D$ and $E$ ). 


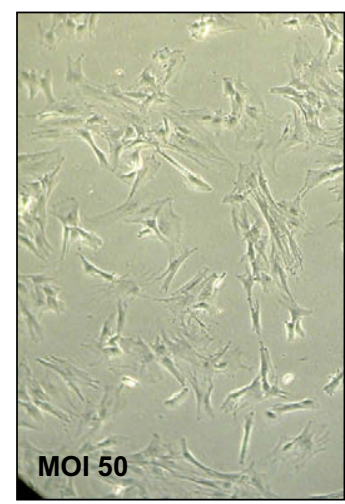

A

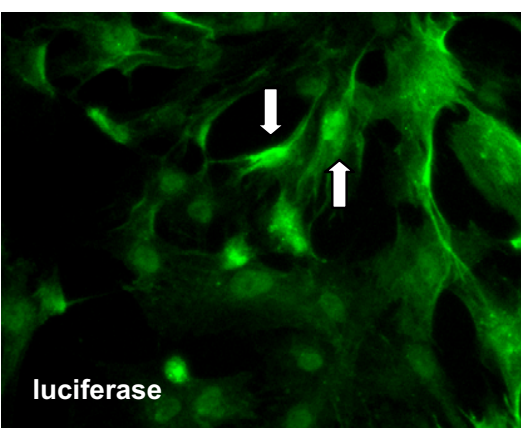

D
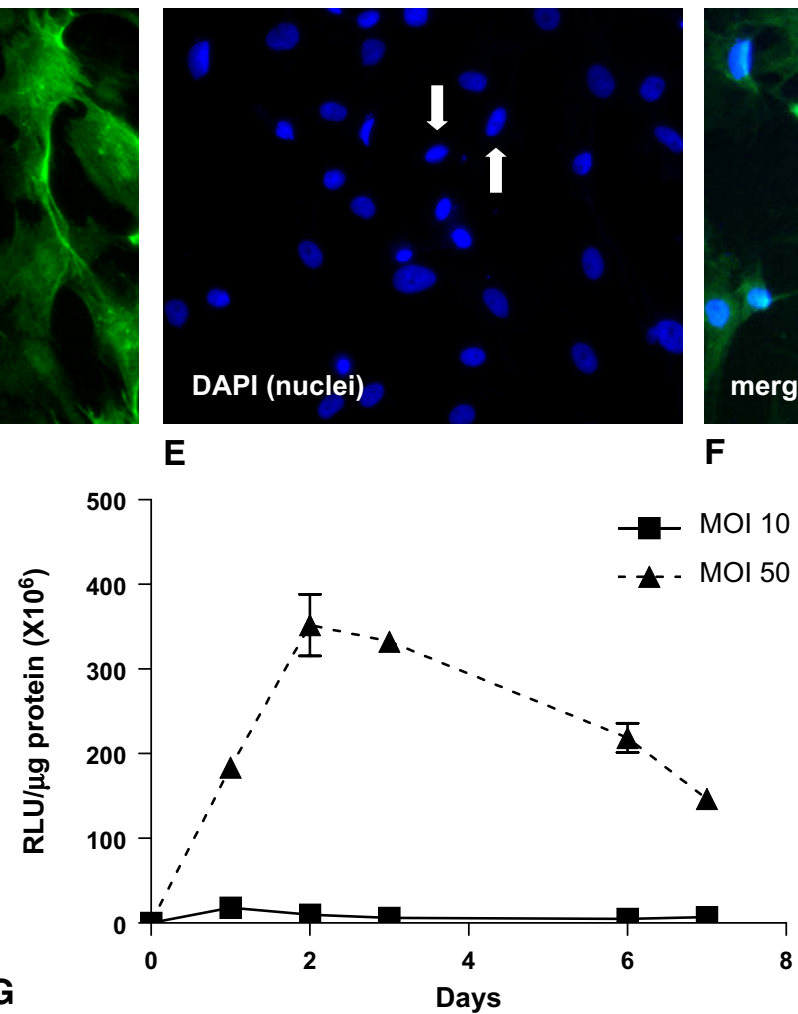

F $0.375 \times 10^{6}$
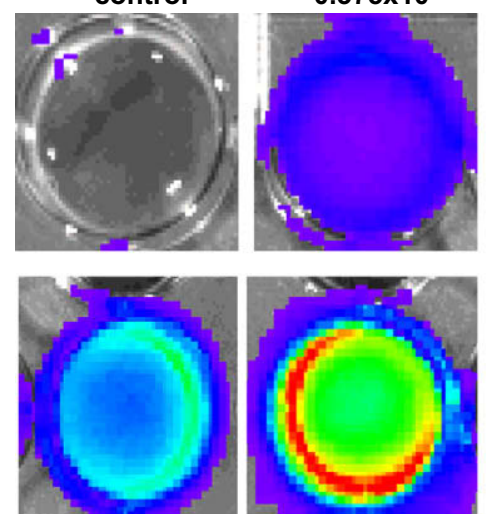

H $\quad 1.5 \times 10^{6}$

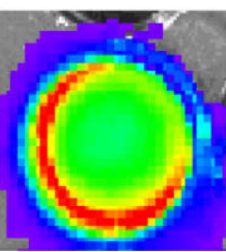

$3.0 \times 10^{6}$
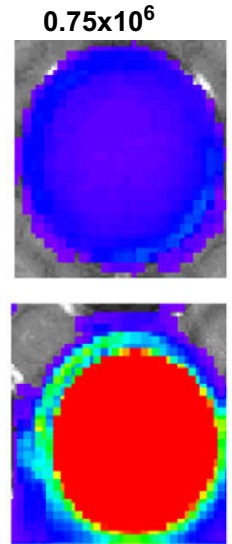

$6.0 \times 10^{6}$
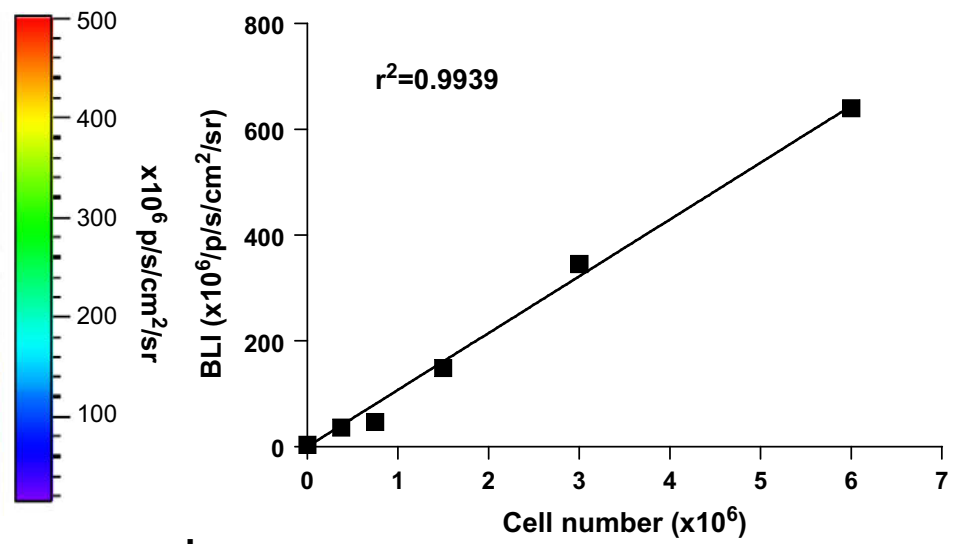

I
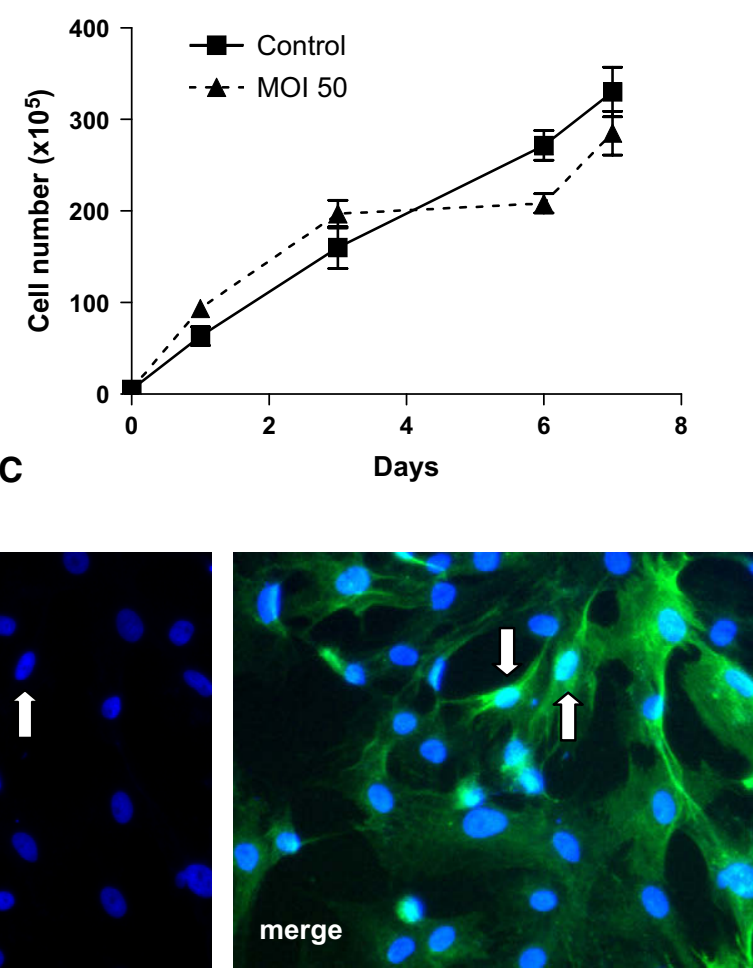


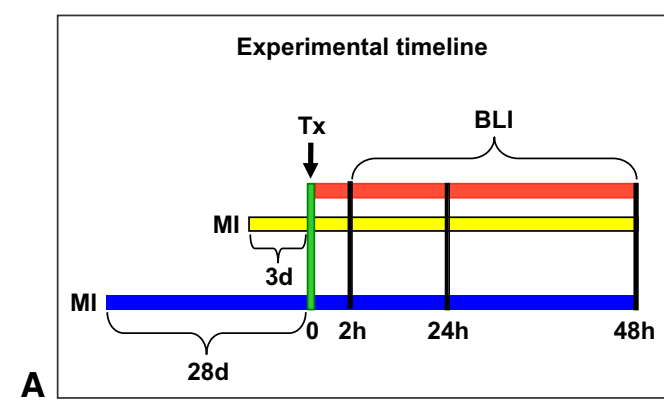

IA
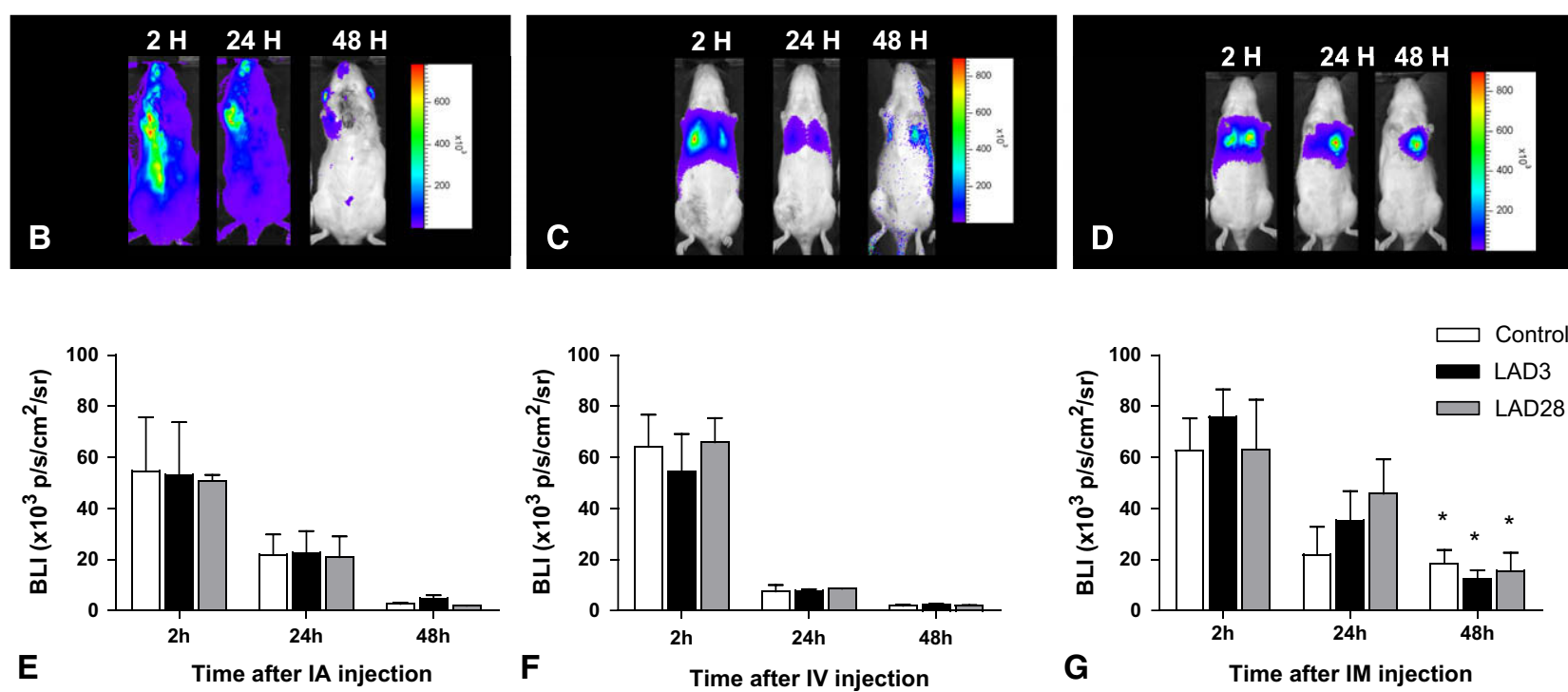

FIGURE 2. In vivo bioluminescence imaging. A, Experimental design. Bone marrow stromal cells transfected with firefly luciferase reporter gene were implanted $(T x)$ into rats after sham operation (Control) or at 3 days (LAD3) or 28 days (LAD28) after coronary artery ligation (MI) by means of 1 of 3 routes: intra-aortic (IA), intravenous (IV), or intramyocardial $(I M)$. Sample sizes for each of the resulting 9 groups are indicated. Whole-body bioluminescence imaging $(B L I)$ was performed at 2, 24, and 48 hours after implantation. Imaging of explanted organs and real-time polymerase chain reaction analysis were performed subsequently at 48 hours. B-D, Representative bioluminescence images obtained at 2, 24, and 48 hours after cell implantation by means of intra-aortic (B), intravenous (C), or intramyocardial (D) injection in control rats (after the sham operation). E-G, Corresponding bioluminescence counts (BLI) integrated over the whole body after cell implantation at LAD3 or LAD28 or in control animals. N = 4 (LAD3-IA, LAD28-IA, LAD28-IV, LAD28-IM), $\mathrm{n}=5$ (Control-IA and Control-IM), and $\mathrm{n}=6$ (Control-IV, LAD3-IV, LAD3-IM) per group. $* P<.05$ versus the corresponding intra-aortic and intravenous groups.

\section{DISCUSSION}

Although preclinical cell therapy produced promising improvements in ventricular function, clinical trials have been less encouraging. For example, cells (bone marrow and peripheral stem cells or skeletal myoblasts) injected intravenously and into coronary arteries early or late after an $\mathrm{MI}^{6-9}$ or into a chronic infarct during coronary bypass surgery in patients with a nonbypassable region ${ }^{10,11}$ produced mixed functional effects. Although the benefits (improved heart function) and the risks (remote organ engraftment or restenosis) of cell therapy are largely determined by the extent of retention of the implanted cells immediately after injection, current approaches to track implanted cells (male cell implantation into female recipients, green fluorescent protein or $\beta$-galactosidase gene labeling, and membrane labeling) exclude the possibility of in vivo cell tracking because they must be performed at the end of the study. The current study describes a molecular imaging technique that provides the

FIGURE 1. In vitro studies. A and B, Cell morphology of cultured bone marrow stromal cells transfected with firefly luciferase gene at multiplicity of infection (MOI) 50 (A) or vector (B). C, Cell proliferation (cell number) in vector-transfected (Control) or MOI 50 colonies. D-F, Immunohistochemical staining (luciferase $=$ green, $\mathrm{D} ; 4^{\prime}$-6-diamidino-2-phenylindole dihydrochloride $[D A P I]=$ blue, E) showing luciferase protein expression in the transfected cells (arrows in D-F). (Original magnification $200 \times$ in D-F.) G, Luciferase activity in cell lysates transfected with viral vector at MOI 10 or 50 for 7 days after transfection. $R L U$, relative light units. $\mathrm{H}$ and I, Correlation between transfected cell number and luciferase activity $(B L I) . \mathrm{N}=3$ per group in $\mathrm{C}$ and $\mathrm{G} . r^{2}=0.99$. 


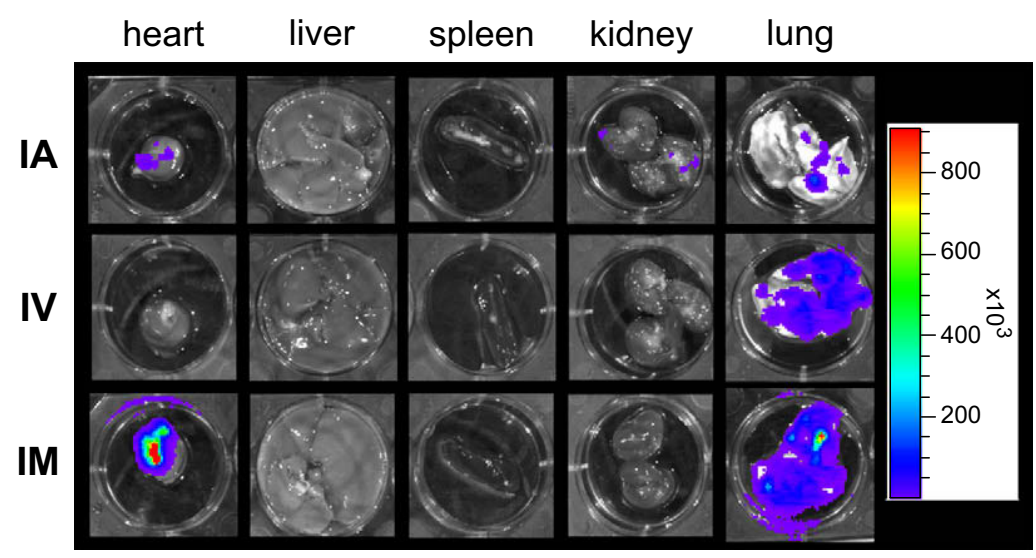

A
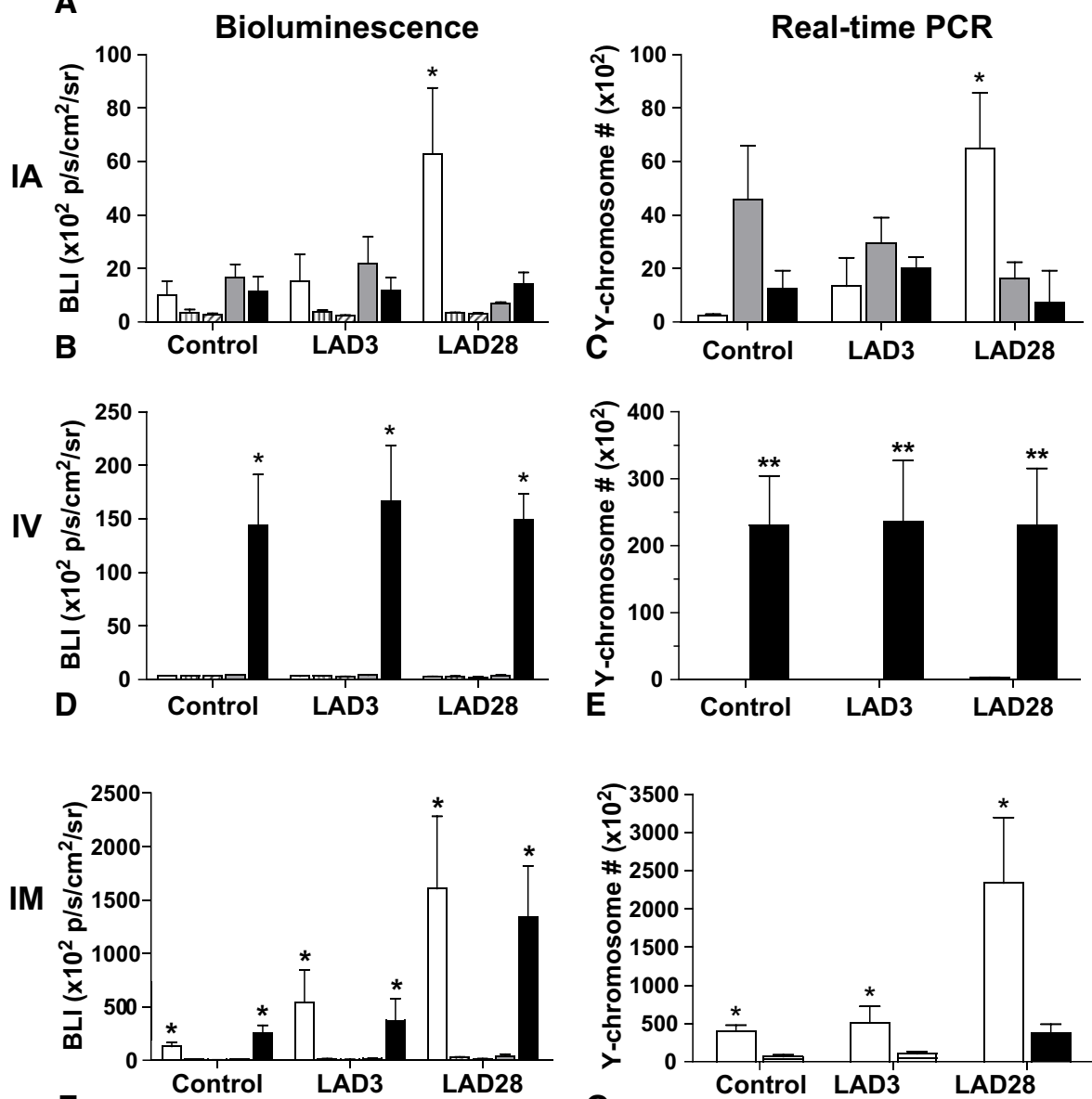

$\mathbf{F}$

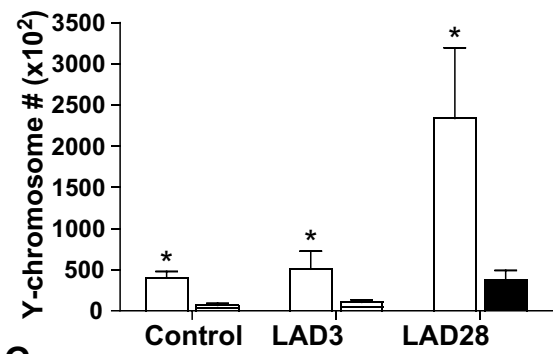

G

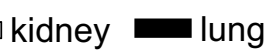

FIGURE 3. Ex vivo bioluminescence imaging. A, Representative bioluminescence imaging in explanted tissues 48 hours after bone marrow stromal cells transfected with firefly luciferase gene were implanted into rats by means of intra-aortic $(I A)$, intravenous $(I V)$, or intramyocardial $(I M)$ delivery at 28 days after coronary artery ligation. B-G, Integrated bioluminescence counts $(B L I)$ and corresponding real-time polymerase chain reaction $(P C R)$ measures $(Y$ chromosome \#) in each tissue and each group at 48 hours after cell delivery performed after the sham operation (Control) or at 3 (LAD3) or 28 (LAD28) days after ligation. Data for liver, spleen, or kidney are omitted where no donor cells were detected. N = 4 (LAD3-IA, LAD28-IA, LAD28-IV, LAD28-IM), $n=5$ (Control-IA and Control-IM), and $\mathrm{n}=6$ (Control-IV, LAD3-IV, LAD3-IM) per group. $* P<.05$ versus the corresponding control and LAD3 groups in $\mathrm{B}$ and $\mathrm{C} . * P<.05$ and $* * P<.001$ versus other organs in $\mathrm{D}$ and $\mathrm{E} . * P<.05$ versus liver, spleen, and kidney in $\mathrm{F} . * P<.05$ versus lung in G. 
A
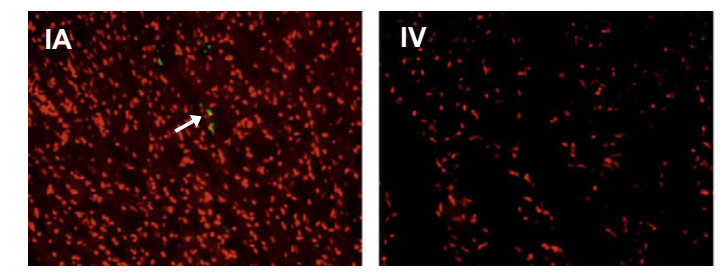

green=luciferase; red=PI (nuclei)
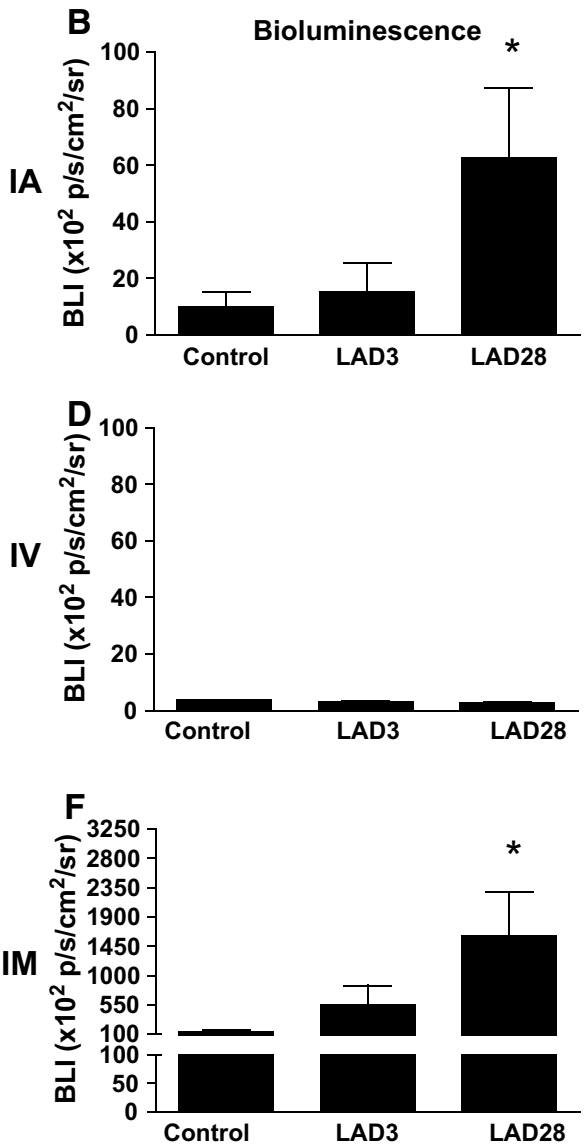

C

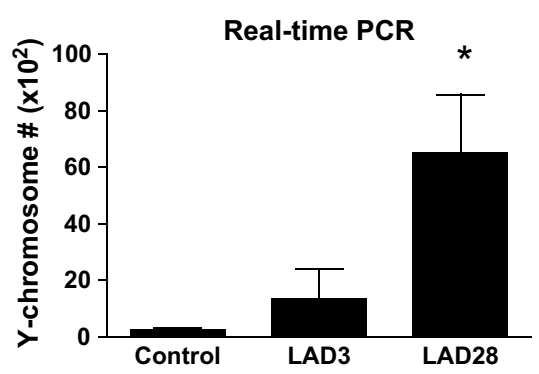

E

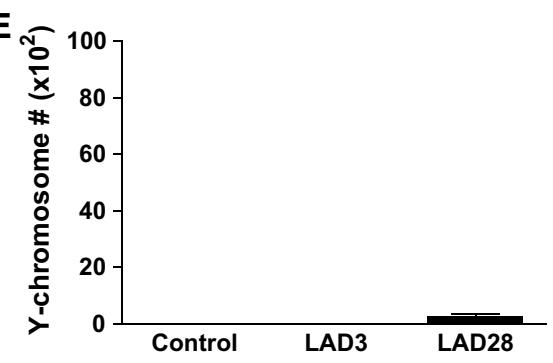

FIGURE 4. Cell retention in the explanted heart. A, Representative micrographs (original magnification $200 \times$ ) illustrating immunostaining for luciferase (green, examples of positive cells indicated by arrows) and propidium iodide ( $P I$; nuclei, red) in heart sections at 48 hours after cells transfected with a firefly luciferase reporter gene were implanted (through intra-aortic [IA], intravenous [IV], or intramyocardial [IM] injection) into rats that had undergone coronary artery ligation 3 days prior. $\mathrm{B}-\mathrm{G}$, Comparison of cell retention (bioluminescence imaging [BLI] and real-time polymerase chain reaction [PCR; $Y$ chromosome \#]) in explanted heart tissue at 48 hours after cell implantation by means of intra-aortic (B and C), intravenous (D and E), or intramyocardial (F and G) injection in rats after the sham operation (Control) or at 3 (LAD3) or 28 (LAD28) days after coronary artery ligation. N = 4 (LAD3-IA, LAD28-IA, LAD28-IV, LAD28$\mathrm{IM}), \mathrm{n}=5$ (Control-IA and Control-IM), and $\mathrm{n}=6$ (Control-IV, LAD3-IV, LAD3-IM) per group. ${ }^{*} P<.05$ versus control and LAD3 groups in B, C, and G. $* P<.05$ versus the control group in $\mathrm{F}$.

opportunity to obtain a clear understanding of implanted cell fate, which is necessary to improve the clinical outcomes of cell therapy.

We used noninvasive optical imaging to track and compare the quantitative distribution of viable BMSCs implanted early or late after an MI by each of 3 clinically relevant implantation routes. Within 48 hours of delivery, the majority of implanted cells were localized in the extracardiac organs. This widespread nontargeted distribution illustrates the importance of tracking cell fate after delivery and the need for novel techniques to increase cell retention. This study is the first to provide a quantitative comparison of live cell imaging and in vitro assessments of cell retention (real-time PCR and immunohistochemistry). Correspondence with the other approaches was excellent, but only imaging permits a serial evaluation in the same animal.

Intracoronary injection is the most common clinical route for cell delivery to the damaged myocardium early after an MI; however, conflicting results from clinical trials stimulated investigations into the biodistribution of cells delivered into 
the coronary arteries. After intra-aortic delivery in the current study, we observed a diffuse systemic distribution of BMSCs that were quickly removed (approximately $90 \%$ by 48 hours by means of bioluminescence imaging of live cells). The remaining cells localized mainly in the heart, lungs, and kidney, with greater cardiac retention when the cells were implanted into a chronic rather than an acute infarct. These data are in agreement with results from a swine study that used radiolabeled human peripheral blood mononuclear cells, ${ }^{20}$ whereas a human study monitoring the distribution of $18 \mathrm{~F}$-fluorodeoxyglucose-labeled bone marrow cells showed limited radioactivity in the heart, with most activity localized to the liver and spleen. ${ }^{21}$ Intracoronary implantation appears to support only limited cell engraftment in the heart immediately after implantation. Cell delivery to the myocardium could also be confirmed by transfecting donor cells with thymidine kinase, which can be tracked by means of noninvasive positron emission tomographic imaging.

After an MI, stem cells are mobilized from the bone marrow into the circulation and eventually home to damaged myocardial tissue. ${ }^{22}$ Increasing the number of circulating stem cells (by means of intravenous implantation) should therefore increase the number of bone marrow cells at the site of injury. Interestingly, Aicher and colleagues ${ }^{23}$ showed that few radiolabeled endothelial progenitor cells homed to the myocardium after intravenous delivery; most were retained in the liver, spleen, and kidney. In the current study intravenously implanted BMSCs homed to the heart, lungs, and kidney, but retention in the heart was minimal at 48 hours after implantation. More than $80 \%$ of intravenously implanted cells were removed by the host within 24 hours after implantation, and only approximately $5 \%$ survived by 48 hours, at which time those remaining resided almost exclusively in the lungs.

Intramyocardial injection has been used to deliver cells directly into the chronically infarcted myocardium during cardiac surgery. ${ }^{10,24}$ Compared with other routes, intramyocardial delivery permits accurate implantation of a larger number of cells and precise targeting of infarct and border regions; however, it is highly invasive. Here we delivered the cells into the infarct by means of direct injection through a thoracotomy. Other investigators have alternatively delivered cells directly into the heart through a catheter through the endocardium or coronary vein. That approach avoids the need for a thoracotomy but increases the potential for damage to the cells as they pass through a long narrow catheter.

We recently reported that $50 \%$ of cells escaped from the heart within 1 hour of intramyocardial injection. ${ }^{25}$ Our current study demonstrates a similar progressive decrease in implanted cell numbers: delivery into normal (sham-operated) or acutely infarcted myocardium resulted in 50\% cell survival in the heart at 2 hours (unpublished data) but greater retention at 48 hours when the cells were injected into a ma- ture infarct. Although we found that some implanted cells leaked from the implanted area into the venous system and were retained in the lungs, intramyocardial delivery produced the greatest cell retention in the heart among the 3 approaches examined. These data are in agreement with previous reports that intramyocardial implantation maximized cell retention in the myocardium compared with other cell delivery routes. ${ }^{26}$

Because clinical trials have implanted cells into patients both early and late after an MI, ${ }^{6-9}$ the timing of cell delivery is another important consideration for cell therapy. This study found improved cell retention after intra-aortic or intramyocardial implantation into hearts with mature rather than acute infarcts, supporting the use of surgical implantation to improve ventricular function. The increase in cell retention might have been due to enhanced BMSC engraftment resulting from progressive neovascularity in the mature infarct, and a decrease in the acute inflammatory response that might have eliminated many of the cells in the acute infarct. The physical integrity of the fibrous scar might also limit cell extravasation and lead to greater cell retention in mature infarcts, particularly during surgical delivery.

Of course, extending our current results to a clinical scenario involves certain limitations. First, the rat model of permanent LAD ligation might not directly mimic the clinical situation in which patients receive percutaneous reperfusion after an MI. However, ligation of a single artery in the rats produced an extensive infarct similar to those seen in patients with diffuse coronary artery disease who are considered for cell transplantation or referred for coronary bypass surgery. Also, because the small size of the animals prevented intracoronary cell delivery, we used intra-aortic injection to simulate intracoronary implantation. Finally, the potential for injected BMSCs to occlude narrow myocardial capillaries should be assessed in light of reports that the intracoronary delivery of bone marrow-derived cells can induce various degrees of myocardial ischemic injury. $^{27}$

Tracked by means of noninvasive molecular imaging, implanted BMSCs exhibited limited cardiac retention and diffuse remote organ distribution within 48 hours of injection. Future studies will focus on new techniques (for example, gene enhancements and addition of biodegradable biomaterials) to improve cell retention, both to increase the efficacy of cell therapy and to decrease any potential adverse effects of the implanted cells.

We thank Heather McDonald Kinkaid for her assistance with manuscript preparation and editing.

\section{References}

1. Kocher AA, Schuster MD, Szabolcs MJ, Takuma S, Burkhoff D, Wang J, et al. Neovascularization of ischemic myocardium by human bone-marrow derived angioblasts prevents cardiomyocyte apoptosis, reduces remodelling, and improves cardiac function. Nat Med. 2001;7:430-6. 
2. Tomita S, Li RK, Weisel RD, Mickle DA, Kim EJ, Sakai T, et al. Autologous transplantation of bone marrow cells improves damaged heart function. Circulation. 1999;100(suppl):II247-56.

3. Kamihata H, Matsubara H, Nishiue T, Fujiyama S, Tsutsumi Y, Ozono R, et al. Implantation of bone marrow mononuclear cells into ischemic myocardium enhances collateral perfusion and regional function via side supply of angioblasts, angiogenic ligands, and cytokines. Circulation. 2001;104:1046-52.

4. Kawamoto A, Gwon HC, Iwaguro H, Yamaguchi JI, Uchida S, Masuda H, et al. Therapeutic potential of ex vivo expanded endothelial progenitor cells for myocardial ischemia. Circulation. 2001;103:634-7.

5. Hodgson DM, Behfar A, Zingman LV, Kane GC, Perez-Terzic C, Alekseev AE, et al. Stable benefit of embryonic stem cell therapy in myocardial infarction. Am J Physiol Heart Circ Physiol. 2004;287:H471-9.

6. Wollert KC, Meyer GP, Lotz J, Ringes-Lichtenberg S, Lippolt P, Breidenbach $\mathrm{C}$, et al. Intracoronary autologous bone-marrow cell transfer after myocardial infarction: the BOOST randomised controlled clinical trial. Lancet. 2004;364:141-6.

7. Assmus B, Honold J, Schachinger V, Britten MB, Fischer-Rasokat U, Lehmann R, et al. Transcoronary transplantation of progenitor cells after myocardial infarction. $N$ Engl J Med. 2006;355:1222-32.

8. Lunde K, Solheim S, Aakhus S, Arnesen H, Abdelnoor M, Egeland T, et al. Intracoronary injection of mononuclear bone marrow cells in acute myocardial infarction. N Engl J Med. 2006;355:1199-209.

9. Schachinger V, Erbs S, Elsasser A, Haberbosch W, Hambrecht R, Holschermann H, et al. Intracoronary bone marrow-derived progenitor cells in acute myocardial infarction. $N$ Engl J Med. 2006;355:1210-21.

10. Stamm C, Kleine HD, Choi YH, Dunkelmann S, Lauffs JA, Lorenzen B, et al. Intramyocardial delivery of CD133+ bone marrow cells and coronary artery bypass grafting for chronic ischemic heart disease: safety and efficacy studies. $J$ Thorac Cardiovasc Surg. 2007;133:717-25.

11. Menasche P, Alfieri O, Janssens S, McKenna W, Reichenspurner H, Trinquart L, et al. The Myoblast Autologous Grafting in Ischemic Cardiomyopathy (MAGIC) trial: first randomized placebo-controlled study of myoblast transplantation. Circulation. 2008;117:1189-200.

12. Amado LC, Schuleri KH, Saliaris AP, Boyle AJ, Helm R, Oskouei B, et al. Multimodality noninvasive imaging demonstrates in vivo cardiac regeneration after mesenchymal stem cell therapy. J Am Coll Cardiol. 2006;48:2116-24.

13. Wu JC, Chen IY, Sundaresan G, Min JJ, De A, Qiao JH, et al. Molecular imaging of cardiac cell transplantation in living animals using optical bioluminescence and positron emission tomography. Circulation. 2003;108:1302-5.

14. Cao F, Lin S, Xie X, Ray P, Patel M, Zhang X, et al. In vivo visualization of embryonic stem cell survival, proliferation, and migration after cardiac delivery. Circulation. 2006;113:1005-14.
15. Li Z, Wu JC, Sheikh AY, Kraft D, Cao F, Xie X, et al. Differentiation, survival, and function of embryonic stem cell derived endothelial cells for ischemic heart disease. Circulation. 2007;116(suppl):I46-54.

16. Fazel S, Chen L, Weisel RD, Angoulvant D, Seneviratne C, Fazel A, et al. Cell transplantation preserves cardiac function after infarction by infarct stabilization: augmentation by stem cell factor. J Thorac Cardiovasc Surg. 2005;130:1310.

17. Yau TM, Kim C, Li G, Zhang Y, Fazel S, Spiegelstein D, et al. Enhanced angiogenesis with multimodal cell-based gene therapy. Ann Thorac Surg. 2007;83: 1110-9.

18. Zhang H, Fazel S, Tian H, Mickle DA, Weisel RD, Fujii T, et al. Increasing donor age adversely impacts beneficial effects of bone marrow but not smooth muscle myocardial cell therapy. Am J Physiol Heart Circ Physiol. 2005;289. H2089-96.

19. Chen SL, Fang WW, Ye F, Liu YH, Qian J, Shan SJ, et al. Effect on left ventricular function of intracoronary transplantation of autologous bone marrow mesenchymal stem cell in patients with acute myocardial infarction. Am J Cardiol. 2004 94:92-5.

20. Freyman T, Polin G, Osman H, Crary J, Lu M, Cheng L, et al. A quantitative, randomized study evaluating three routes of mesenchymal stem cell delivery following myocardial infarction. Eur Heart J. 2006;27:1114-22.

21. Hofmann M, Wollert KC, Meyer GP, Menke A, Arseniev L, Hertenstein B, et al. Monitoring of bone marrow cell homing into the infarcted human myocardium. Circulation. 2005;111:2198-202.

22. Bittira B, Shum-Tim D, Al-Khaldi A, Chiu RC. Mobilization and homing of bone marrow stromal cells in myocardial infarction. Eur J Cardiothorac Surg. 2003;24 393-8.

23. Aicher A, Brenner W, Zuhayra M, Badorff C, Massoudi S, Assmus B, et al. Assessment of the tissue distribution of transplanted human endothelial progenitor cells by radioactive labeling. Circulation. 2003;107:2134-9.

24. Tran N, Franken PR, Maskali F, Nloga J, Maureira P, Poussier S, et al. Intramyocardial implantation of bone marrow-derived stem cells enhances perfusion in chronic myocardial infarction: dependency on initial perfusion depth and follow-up assessed by gated pinhole SPECT. J Nucl Med. 2007;48:405-12.

25. Yasuda T, Weisel RD, Kiani C, Mickle DA, Maganti M, Li RK. Quantitative analysis of survival of transplanted smooth muscle cells with real-time polymerase chain reaction. J Thorac Cardiovasc Surg. 2005;129:904-11.

26. Hou D, Youssef EA-S, Brinton TJ, Zhang P, Rogers P, Price ET, et al. Radiolabeled cell distribution after intramyocardial, intracoronary, and interstitial retrograde coronary venous delivery: implications for current clinical trials Circulation. 2005;112(suppl):I150-6.

27. Vulliet P, Greeley M, Halloran S, MacDonald K, Kittleson M. Intra-coronary arterial injection of mesenchymal stromal cells and microinfarction in dogs. Lancet. 2004;363:783-4. 


\section{MATERIALS AND METHODS \\ Myocardial Infarction and Cell Transplantation}

Female rats were anesthetized with isoflurane (2\%) during mechanical ventilation. MI was induced through a thoracotomy by means of occlusion of the LAD. The animals were randomly separated into 6 groups. Each rat received $3 \times 10^{6}$ transfected BMSCs (10-20 $\mu \mathrm{m}$ in diameter) in $100 \mu \mathrm{L}$ of serum-free medium at 1 of 2 time points (ie, 3 or 28 days after LAD ligation [LAD3 and LAD28, respectively]) delivered through 1 of 3 routes (detailed below): intra-aortic, intravenous, or intramyocardial injection. Three additional groups without LAD ligation (sham controls) also received BMSCs by means of intra-aortic, intravenous, or intramyocardial injection (9 groups total).

Intramyocardial implantation was performed after a thoracotomy, as previously described. ${ }^{\mathrm{E} 1}$ Cells were injected into 3 locations within the infarct to ensure adequate distribution through the infarct and border regions. In the intravenous implantation group cells (in serum-free medium) were injected into the femoral vein through a hind-limb incision. In the intra-aortic implantation group (injection into the ascending aorta with the distal aorta occluded to simulate intracoronary implantation), the right neck and upper sternal area were incised to expose the common carotid artery, brachiocephalic trunk, aortic arch, and ascending aorta. 5-0 Silk ties were placed under the aortic arch between the left common carotid artery and the brachiocephalic trunk, under the right common carotid artery, and under the right subclavian artery. A 24gauge, 0.75-inch intravenous catheter (BD Biosciences, Mississauga, Ontario, Canada) was inserted from the common carotid artery into the aortic root above the aortic valve. The aortic arch, the right common carotid artery, and the right subclavian artery were occluded with the silk ties for 5 seconds during cell injection to ensure the cells were delivered into the coronary arteries. This technique delivers cells into the coronary arteries but differs from the clinical scenario in 2 aspects: the anterior coronary artery remains occluded (whereas it is usually opened after an infarction), and some cells remain in the aortic root (whereas the clinical double-balloon technique reduces cell leakage into the aorta). The neck and upper sternal incisions were closed by suturing the muscle layers and skin with 3-0 Vicryl sutures (Ethicon, Inc, Somerville, NJ).

\section{Reference}

E1. Tomita S, Li RK, Weisel RD, Mickle DA, Kim EJ, Sakai T, et al. Autologous transplantation of bone marrow cells improves damaged heart function. Circulation. 1999;100(suppl):II247-56. 